\title{
Algunas Determinaciones del Pregnandiol Urinario en el Aborto Amenazante
}

TRABAJO INEDITO PRESENTADO POR EL DOCTOR

Eumoro Castillo Veca

PARA PROFESOR AGREGADO DE LA FACULTAD DE MEDICINA

(Clínica Obstetrica)

1954

\section{INTRODUCCION}

El presente trabajo tiene por objeto hacer algunas "determinaciones del pregnandiol urinario en enfermas con sintomas evidentes de aborto amenazante". o bien, en enfermas en cuva historia anamnésica se encuentre el antecedente de uno o más abortos anteriores, asi como también de partos prematuros y que, ni en los primeros ni en los últimos se hava encontrado una causa bien determinada. En lo posible, se han escogido pacientes con antecedente Scrológico Negativo y de constitucion topográfica anatómica, en cuanto a pelvis se refiere, normales. Es de lógica que, a una enferma con una desviación uterina o con un tumor pélvico, no se puede achacar el aborto a deficiencia de Progesterona o de cualquiera otra hormona, pues la causa es verdaderamente objetiva. Tampoco lo sería la gestante cuya historia clínica presentara unas reacciones de Wassermann, de Kahn o de Mazzini francamente positivas. Pero también es verdad que pueden presentarse preñeces patolegicas de tal complejidad, que a los datos serol feros y anatómicus se agrega una considerable baja del pregnandiol urinarios en dicho caso, necesartamente lia determinación cuantitativa de su chaminación urinaria, contribuirá notablemente a sel clicar tratamiento. Por los anterioses considerandes y pueso que la mencionada deteminación está, laes

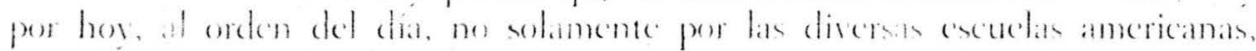

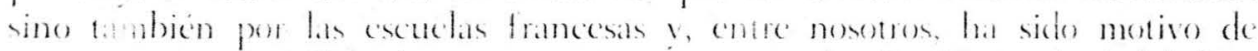
preocupacion cientifica de maestres insignes como los Profesores fosé del Car 
men Acosta y Arturo Aparicio y para no citar más, emprendimos con entusiasmo la búsqueda de las enfermas en cuestión, principalmente entre la clientela particular, pues bien sabemos por desgracia que a los centros hospitalarios llegan las enfermas en situaciones alarmantes, cuando el aborto es matcrialmente im posible de detener $y$, en la mayoría de las veces, cuando el aborto ya se ha iniciado y solamente se busca al médico obstetra para la extracción de restos ovulares.

Sin embargo, agrupamos cierto número de observaciones clínicas para la determinación del pregnandiol urinario, ejecutando dicho examen en varias ocasiones a la misma enferma, para poder resumir algunas conclusiones, que aunque no son ni pueden ser la última palabra en tan intrincado problema, por lo menos son la iniciación para futuras y más completas controversias científicas.

La técnica de Laboratorio para la determinación del pregnandiol urinario es compleja y dispendiosa, pero no difícil. A pesar de todo y con el objeto do no incurrir en error técnico alguno, nos asesoramos del muy conocido labora. torista y por demás experto en esta clase de análisis, el doctor César Mendoza. quien siendo el Director del Laboratorio Clínico del Instituto Nacional de Cancerología, nos prestó su valiosa colaboración y nos facilitó el laboratorio del Instituto para la ejecución de todas las determinaciones de pregnandiol contenidas en este trabajo. Para él y para la señorita Marina Rangel, su diligentc ayudante, nuestros agradecimientos muy sinceros.

Hemos prescindido adrede en la elaboración del trabajo, de los pormenores anatómicos, fisiológicos e histoanatómicos del aborto, por considerarlos del dominio del obstetra y además, fuera de lugar.

Solamente consignamos pequeños detalles acerca del origen del pregnandiol por considerarlo básico, la técnica usada por nosotros para su determinación urinaria, las historias clínicas, que solamente su lectura nos dan idea de la importancia en la determinación cuantitativa del metabolismo de la progesterona y por fin, las conclusiones muy escuetas a que hemos podido llegar.

\section{ORIGEN Y PAPEL DE LA PROGESTERONA}

Experimentalmente es sabido que la progestorona secretada por el cuerpo amarillo en el principio del embarazo, más tarde por la placenta cuando ésta se ha formado y en muy pequeña escala por las suprarrenales maternas y hasta por las suprarrenales fetales, se ha llamado con muy buenas razones la "hormona de la preñez por excelencia". Recientemente Colvin es contrarin a esta creencia y es de opinión de que los entusiastas elogios dados y atribuídos a la progesterona y no se justifican. Sin embargo, la fisiologia nos enseña que estal hormona es indispensable para la sobrevida del huevo fecundado y para asegugurar su perfecta nidación, de la misma manera que para garantizar la supervivencia de la preñez misma. En otras palabras, provoca por un laclo, la trans formación del endometrio necesaria a la nidación del huevo permitiendo a éste 
su fijación y nutrición; por otra parte, reduce la excitabilidad de la musculatura lisa del útero y también la contractilidad de la misma. A la hormona luteínica (nombre impropio) debido a su acción biológica, le ha valido el nombre do progesterona.

En la práctica, los buenos resultados obtenidos con la terapéutica de la progesterona en las amenazas de aborto, nos hacen pensar en su efectividad para el mantenimiento de la gestación.

Entre el gran número de trabajos que han aparecido acerca de esta cuestión, destacamos una comunicación concerniente al tratamiento mediante hormona del cuerpo amarillo, con el empleo de la cual, el autor, ha visto nacer un 50\% de niños vivos después de tres abortos y casi $45 \%$ después de cuatro abortos ${ }^{1}$ ).

Al juzgar los resultados del tratamiento del aborto habitual es preciso tomar como punto de partida ciertos datos estadísticos $\left({ }^{2}\right)$ según los cuales las probabilidades de un embarazo normal sin tratamiento hormonal se eleva a:

$78 \%$ después de un aborto.

62\% después de dos abortos consecutivos.

$27 \%$ después de tres abortos consecutivos, y

$6 \%$ después de cuatro abortos consecutivos.

Recordamos a continuación los resultados conseguidos en Inglaterra gracias a la progesterona: en una serie de casos relativamente restringida, los autores comunican resultados favorables en $88 \%$ de los casos después de tres abortos en $60 \%$ después de cuatro abortos. Según la tendencia más reciente de combinar la progesterona con estrógenos, se obtuvo a partir de 1945 el nacimiento global de 16 niños vivos en un grupo de 24 mujeres que habían tenido en el grupo 73 abortos $\left({ }^{4}\right)$. Puede admitirse que en esta clase de tratamiento combinado, la hormona estrógena ejerce un efecto de ahorro de la progesterona, va que se comprueba cada vez más que cuando se utiliza la progesterona aislada mente, es preciso llegar a dosis muy elevadas en ocasiones.

Recordamos también que algunos autores han tenido la impresión de que en ciertos casos, por otra parte raros, el empleo de la progesterona puede precipitar los síntomas del aborto y facilitar el aborto mismo. Como también se creyó que la Foliculina tenía en efecto abortivo; no fue sino muy secundariamente, después de múltiples experiencias terapéuticas, como se ha podido constatar que la foliculina en la mujer no tiene acción abortiva por lo menos en el curso de la preñez normal bien establecida. Se debe a $G$. V an Smith y a su esposa, quienes fueron los primeros en mostrar la acción favorable de la foliculina en ciertas preñeces patológicas y en particular en las amunazas de oborto o en abortos de repetición. Ellos han mostrado igualmente que en estos casos los dosajes hormonales han revelado casi siempre cifras anormalmente bajas de "pregnandiol" y también de estrógenos.

Todas la hormonas, tanto las elaboratas por las glándulas de secreción in terna como aportadas al organismo del exterior, están diseminada en el mísmo. 
según unas leyes generales de distribución. Esal distribución depende dẹ equilibrio de solubilidad y de absorción, y la velocidad del establecimiento de este equilibrio, de la permeabilidad de las diversas membranas y de la velocidad de difusión de las hormonas, cada una de ellas en particular. Las hormonas, lo mismo que otras sustancias, están sometidas a la influencia del metabolismo. Generalmente se destruven con relativa facilidad. Su constante de semidesintegración biológica es la mayoría de las veces, breve, tanto por parte de la eliminación como por parte de la distribución y de la desintegración metabólica. Las reacciones que utiliza el organismo para la desintegración de las hormonas, son totalmente reacciones metabolicas corricntes. Sobre las influencias deshidrogenantes y reductoras que actúan en la desintegración de las hormonas esteroides, como reacciones principales, orienta la fórmula que muestra la desintegración de la progesterona en pregnandiol.

Según: STEEL, MATHEW

Philadelphia.

Lea-Febriger, 1937.

Fhiladelphia

P. 86.

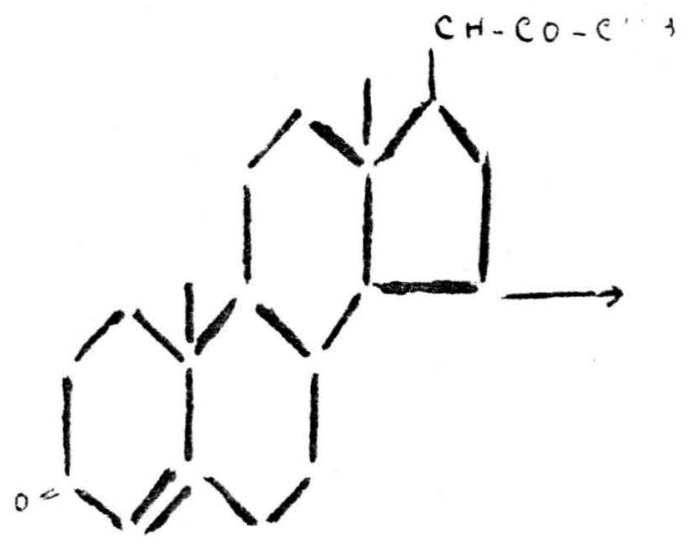

PROGESTEKUNH

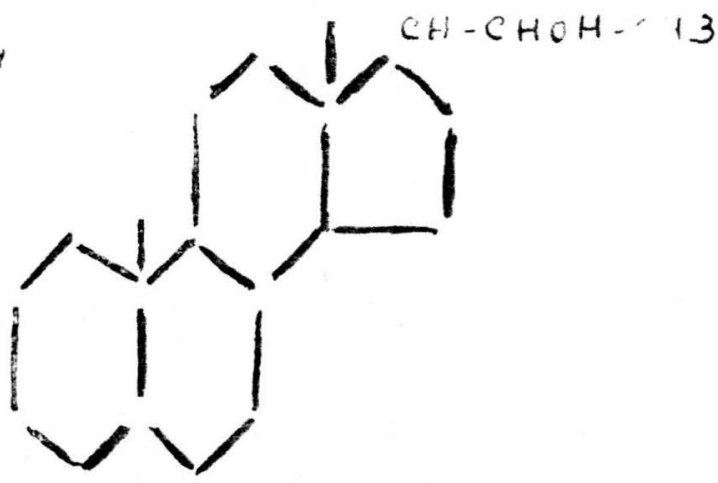


En relación a las hormonas esteriodes repercute desfavorablemente el hecho de que, después de la administración oral, aparecen primeramente en la circulación de la vena porta y con frecuencia, antes de pasar al resto del organismo, experimentan en el hígado una pérdida de actividad, debido a su destruc ción en tal órgano $\left({ }^{6}\right)$.

El Pregnandiol es pues el metabolito de la progesterona, aunque no es el unico. Es biológicamente inactivo, pero su dosificación nos permite avaluar in directamente, con cierta relativa precisión, la producción de progesterona en el organismo. Es pues el pregnandiol un esteroide inactivo biológicamente, derivado de la progesterona. Se encuentra normalmente en la orina de la mujer embarazada ( $\left.{ }^{\top}\right)$. Su designación química es la siguiente:

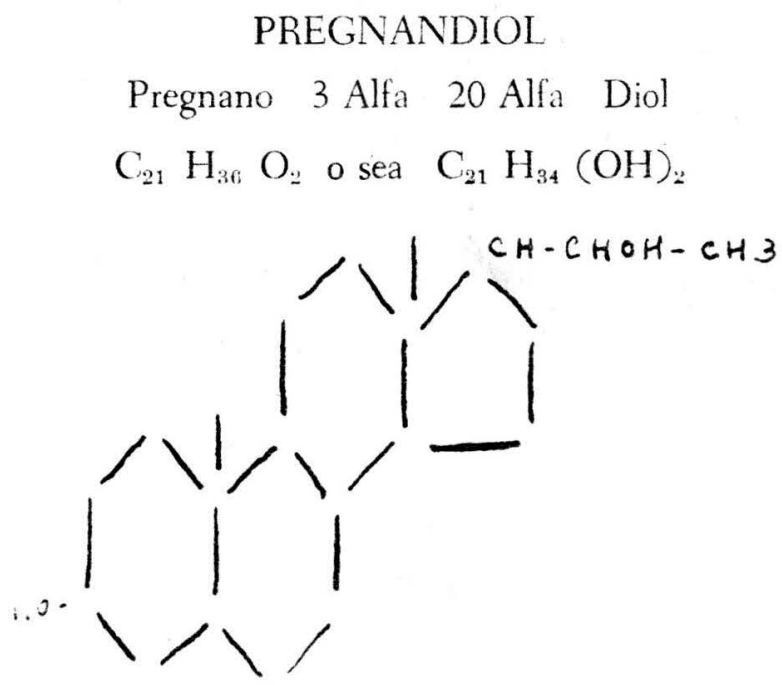

Su eliminación por la orina se hace combinado con el ácido glucorónico. Su inactivación se hace por reducción: Progesterona en Pregnandiol. En los glucuronidatos de pregnandiol, el grupo indoxilo del esteroides se combiana con el grupo aldehido del ácido glucosánico, grupo este último en el que precisamente reside la propiedad reductora. La conjugación de los esteroides con el ácido GLUCURONICO o SULFURICO se realiza en el hígado, puesta tanto la hepatectomía como las lesiones hepáticas por substancias tóxicas impiden el proceso de conjugación.

Los conjugados, a diferencia de los esteroides libres, son altamente hidrosolubles, lo que facilita su eliminación urinaria; es el caso de] pregnandiol, pro ducto de reducción de la progesterona que se excreta en la orina en la forma de glucuronidato de pregnandiol sódico, previa conjugación con el ácido glucuró nico o sulfúrico.

Por lo que hace a la progesterona administrada, Arthur K. Koff ("'), logró cstablecer que cuando sc administran 100 miligramos de progesterona intra 
muscularmente a una embarazada normal, 30 miligramos de pregnandiol son excretados por la orina. Por otra parte, cuando se administran 100 miligramos de progesterona a una embarazada con aborto amenazante y baja secreción d: pregnandiol, solamente 10 miligramos adicionales de pregnandiol son secre tados por la orina. Este experimento demuestra que en el último caso solamente el $10 \%$ de la progesterona administrada se utiliza.

\section{DETERMINACIÓN DEL PREGNANDIOL MUINARIO)}

Desde que se estableció, o mejor aún, desde que se descubrió que la excreción del pregnandiol es una medida de la producción de progesterona, muchos autores se dedicaron a dosificar la cantidad de pregnandiol urinario para sacar como resultado práctico en el ejercicio de la tocología, el pronóstico bueno o malo en cuanto a la supervivencia de la gestación. Browne, Henry y Venning ( ${ }^{8}{ }^{9}$ ), observaron que la mayoría de las pacientes con síntomas de aborto amcnazante, que excretan una cantidad de pregnandiol inferior a la normal, fallan en mantener su preñez. Por otra parte, cuando la excreción de pregnandiol per manece normal, los síntomas de aborto anemazante se atenúan y la gestación reasume su curso normal ( $\left.{ }^{10}\right)$.

Venning fue el primero en describir un método de dosificación químicopráctico del glucuronato de pregnandiol. Es un método ponderable, preciso, pero un poco largo ${ }^{11}$ ).

Gutherman encontró un método más sencillo, un poco más rápido (relativamente), utilizando un método colorimétrico $\left({ }^{12}\right)$.

Nosotros, pensando con Dobriner, quien demostró que el pregnandiol no es más que uno de los metabolitos de descomposición de la progesterona y que existen más, resolvimos no seguir la técnica de Gutherman, sino la técnica de Sommerville-Marrian y Kellar, que parece más precisa, pues investiga más particularmente el pregnandiol o solamente a él. Este es el motivo por el cual las cifras normales son más bajas que las señaladas por Gutherman.

A pesar de todo, y como un motivo especial es también de este trabajo, el de popularizar la dosificación hormonal del pregnandiol urinario entre nosotros, queremos consignar a continuación la célebre técnica de Gutherman, puestu que en las revistas extranjeras que sobre el presente tópico hablan, mencionan muy repetidamente ese Metodo Colorimétrico.

\section{TÉCNCA DE GUTHERMAN}

REACTIVOS:

Tolueno puro. redestibiato.

Acido Clombidrico ( : P.

Solución de Hidroxido de Sodio, décime normal.

Solución de llidroxido de Sodio al $2 \%$ en menanol absolum 
Acetona C. P.

Alcohol Etílico absoluto.

Acido Sulfurico (. P.

TÍCNIC:

Se requiere una muestra de orina de $2+$ horas. Si el examen se va a realizar en el mismo dia en que se termine la recoleccion, no es nesesario usar preser rativo; si no, pueden agregarse 10 c.e de acido clorhidrico por cada litro de orina.

A.-Hidrolisis y Extraccion de Pregnandiol:

19-Se colocan en un balón de 500 c.c., 100 c.c. de orina, 50 c.c. de Te lueno, 10 c.c. de ácido clorhídrico concentrado y 2 o 3 perlas de vidrio.

2.-Se conecta el balón a un condensador de Liebig de 400 a $500 \mathrm{~mm}$. d. largo, en posición vertical. La mezcla se hierve vigorosamente por 15 minutos.

39-El balón y su contenido se enfrían hasta la temperatura ambiente bajo un chorro de agua.

4. - Se pasa la mezcla a un embudo de separación de 500 c.c. y se descarta el líquido inferior (orina).

50-La capa de Tolueno y la emulsión se lavan dos veces con porciones de 15 c.c. de Hidróxido de Sodio N/10 y dos veces más con 15 c.c. de agua destilada.

B.-Precipitación de las Impurezas:

10-El lavado de Tolueno y la emulsión (A, 5) se transfieren a una fiola de Erlenmeyer con dos perlas de vidrio.

20-La mezcla se calienta.

30-Cuando el agua se hava evaporado y la mezcla de Tolueno hierva sua vemente, se agregan 10 c.c. de la solución de Hidróxido de Sodio al $2 \%$.

4\%-Se evapora la mezcla hasta que aparezca un precipitado granular. Queda aproximadamente la mitad del volumen del Tolueno.

5.- La mezcla de Tolueno se filtra, cuando está todavía caliente, en un filtro de vidrio (porosidad media Pyrex) con vacío suave. Si el filtra do tiene un color anaranjado, rosado o carmelita, se repiten las operaciones B3, B4 y B5, hasta que el filtrado sea amarillo, o amarillo limon.

60-Se hav d precipitado B con 15 c.e. de Tolueno caliente.

70-Los filtrados B5 y B6 : meztan y se craporan hasta secarlos comple tamente: se usa una corriente de aire suave para desalojar las ultima huellar de foluene. Esto previene la ignicion del residuo. 
C.-Precipitación del Pregnandiol:

10-Se agregan 5 c.c. de acetona al residuo B7 y la mezcla se calienta hasta que la solución sea completa.

$2^{\circ}$ - - Sc agregan, lentamente, 20 c.c. de una solución de Hidróxido de Sodio $\mathrm{N} / 10$. La mezcla se hierve por tres minutos.

$3^{\circ}$ - La fiola se coloca en el refrigerador ( 5 grados C.) por una hora.

\section{D.-Separación del Pregnandiol:}

$1^{\circ}$ - Se filtra la mezcla C3 en un filtro de vidrio (porosidad media Pyrex), con suave succión.

2 - El precipitado Dl se lava con 15 c.c. de agua destilada.

3 - La fiola se cambia y se pasan 10 c.c. de alcohol absoluto caliente a través del filtro de vidrio para disolver el precipitado.

40. El filtrado de alcohol D3, se evapora hasta la sequedad en el frasco receptor.

\section{E.-Desarrollo del Color y Medida Cuantitativa:}

10--Se agregan 10 c.c. de ácido sulfúrico concentrado al residuo D4.

2 ? - Se espera una hora para el desarrollo del color, agitando de vez en cuando.

30.-Una fracción determinada de la fracción E2, se diluye hasta un volumen de 5 c.c. con ácido sulfúrico concentrado, en un tubo seco del corímetro, y se mezcla la solución.

40- Se lee el color en fotocolorímetro a $430 \mathrm{Mp}$, usando como blank ácido sulfúrico concentrado.

50-La cantidad de pregnandiol contenida en la alícuota de orina usada se obtiene de una curva de calibración. Para calcular la cantidad de pregnandiol eliminado en 24 horas debe tenerse en cuenta la dilución inicial y el volumen urinario excretado en dicho período de tiempo.

\section{CURVA DE CALIBRACIÓN}

Sc extrajo pregnandiol de orina humana de embarazo, siguiendo exactamente el procedimiento de Gutherman, por dificultad de la obtención de pregnandiol cristalino puro.

Del pregnandiol asi obtenido, se disolvieron $10 \mathrm{mlg}$. en 100 c.c. de alcohol absoluto. Te esta solución se tom ron cantidades de 0,1 c.c.; o, $2 ; 0,3 ; 1,+; 0,5$; 0,$6 ; 0,7 ; 0,8 ; 0,9 ; 1,00$ c.c., que contienen respectivamente: 10, 20 microgramos, etc., hasta 100 microgramos (una décima de miligramo).

So evaporan al Bano María y después de enfriar se agrega a cada tubo 5 c.c. de ácido sulfúrico concentrado; se espera una hora y se lee el color en un dectrofotometro de Finer con filtro de $+25 \mathrm{M}$ p. 
Se obtienen lecturas en la escala logarítmica del colorímetro, así: $2 ; 4 ; 6 ; 8$; 9,8; 11,8; 15,7; 17,7; 19,6 (Fig. No 1).

Trasladadas estas lecturas a papel milimetrado centesimal como ordenadas, y las concentraciones correspondientes (en microgramos) como abcisas, y unidos luégo los puntos de intersección correspondientes, se obtuvo una línea recta, conforme a la Ley de Beer Lambert. Esta es la que se emplea como gráfica de calibración para la técnica de Gutherman.

Para usar esta gráfica en la determinación cuantitativa del pregnandiol que contiene una muestra de orina, se procede así: Se toma una cantidad determinada de la solución E2, teniendo en cuenta la intensidad de color, y se diluve hasta un volumen de 5 c.c. con ácido sulfúrico concentrado. Se lee al colorímetro y por medio de la curva se determina a qué cantidad de pregnandiol corresponde esa lectura; el resultado se multiplica por la dilución. Así tenemos el pregnandiol correspondiente a los 100 c.c. de orina que se sometieron al procedimiento. Conocido el volumen en 24 horas, es fácil obtener la cantidad de pregnandiol que contiene la muestra total.

Ejemplo: Volumen total en 24 horas: 1.850 c.c.

Se tomaron 0,5 c.c. de la solución E2 cuyo volumen total es de 10 c.c., es decir, la veintiava parte.

Lectura colorimétrica: 15.

Equivalencia de esta lectura en la curva de calibración: 77 microgramos.

Pregnandiol contenido en 100 c.c. de orina: $77 \times 20=1.540$ microgramos $=1,54$ miligramos. $1,54 \times 1,850=28,5 \mathrm{mlg}$. en volumen en 24 horas.

\section{0}

Fórmula: Lec. curva en meg. X Dil. xcc ori 24 horas = mlg. en V 24 h.

\section{0}

Las dificultades técnicas que presenta la realización de esta prueba, pueden evitarse si se observan las precauciones siguientes:

$1^{0}-$ Usar únicamente reactivos concentrados puros.

20-Preparar la solución de hidróxido de sodio en metanol cada semana.

30 - Usar una corriente de aire para prevenir la ignición de los residuos en las etapas B7 y D4.

40.-Usar filtros de vidro calcinado.

Las muestras de orina deben ser de 24 horas y el examen debe hacerse ef mismo día en que se termine la recolección, pues ségún los experimentos hechos por Robert E. Hoyt y colaboradores (12), la exactitud de la prueba no es la misma si se realiza con muestras de orina vicjas (1:3). 


\section{TÉCNICA DE SOMMERVILLE-MARRIAN Y KELLAR}

REACTIVOS:

Acido Clorhídrico Concentrado.

Tolueno.

Alcohol Etílico Absoluto.

Acido Sulfúrico Concentrado.

Soda Normal.

Soda Décimo Normal.

Norit A.

Muestra de orina de 24 horas, medida en c.c., preservada con Tolueno.

A.-Hidrolisis y Extracción del Pregnandiol:

19-A 100 c.c. de orina se le agregan 50 c.c. de Tolueno químicamente puro, libre de Tiofeno, y 10 c.c. de ácido clorhídrico concentrado.

20-Hervir con reflujo durante 10 minutos y enfriar inmediatamente con agua helada.

$3^{\circ}$ - En un embudo de decantación, separar la capa de Tolueno conservando la emulsión, después de agitar y dejar en reposo durante cinco minutos.

4\%-Filtrar el Tolueno en un embudo de Buechner con succión suave.

50.-Traspasar el filtrado a un embudo de decantación.

B.-Purificación del Pregnandiol:

19.-Lavar con 15 c.c. de soda normal, por dos veces, conservando la capa de Tolueno.

2? - Lavar con agua destilada.

30-Pasar el Tolueno a un Erlemayer y evaporar a sequedad a presión reducida.

49.-Disolver el precipitado en alcohol etílico caliente y reducir a un volumen de 5 c.c. por evaporación.

C.-Precipitación del Pregnandiol:

1.-A los 5 c.c. de la solución alcohólica, agregar lentamente, con agitación constante, 20 c.c. de soda décimo normal, manteniendo el tubo en Baño María a $75^{\circ} \mathrm{C}$.

20-Dejar en el Baño 1 minut y llevar a estufa a $37^{\circ} \mathrm{C}$. durante do horas.

30- Filtrar en un cmbudo de mallas de vidrio, con poros de 20 a 60 micras, con succión suve.

4.-Lavar el precipitado del embudo varias veces con agua destilada, para quitar cualquier resto de soda. 
50-Lavar con alcohol caliente en cantidades de 5 c.c. por tres veces, re cogiendo los filtrados que contienen el Pregnandiol.

60-Añadir algunos miligramos de Norit A y hervir al Baño María por 1 minuto.

Filtrar guardando el filtrado.

D.-Desarrollo del Color:

10-Evaporar a sequedad el filtrado y agregar 10 c.c. de ácido sulfúrico concentrado.

20-Dejar a la temperatura ambiente durante 20 minutos, agitando de vez en cuando con una varilla de vidrio.

3.-Leer en el foto-colorímetro con filtro azul, usando un blank de ácido sulfúrico concentrado.

4은 - Los valores en miligramos por ciento, se obtiene de la curva calibrada con concentraciones conocidas de pregnandiol puro.

NOTA: Para obtener el valor en 24 horas, se sigue la siguiente fórmula:

Pregnandiol en miligramos por $\% \times$ c.c. de orina de 24 horas.

A este dato aritmético se puede agregar en la práctica $2 \mathrm{~mm}$. más como coeficiente de pérdida que pudiera quedar en el recipiente en donde se recibe la orina.

VENTAJAS:

Es absolutamente sencilla en su ejecución y menos laboriosa que la Gutherman.

No necesita de aparato especial complicado, ni reactivos difíciles de conseguir.

Es muy reproductible dentro de niveles clínicos.

La precipitación del pregnandiol a $75^{\circ} \mathrm{C}$., seguida de enfriamiento lento es más completa y más específica que a temperatura ambiente en nevera (Gutherman).

La purificación con Norit A le quita los extractos cromógenos que pueden alterar la reacción de color dando valores más altos que los verdaderos. 


\section{VALORES NORMALES DE PREGNANDIOL EN EL EMBARAZO}

Según Cantarwow - (Clinical Biochemestry, 1950)

Semanas de Amenorrea

$\begin{array}{rrr}2 & 2-10 & 6 \\ 4 & 5-15 & 10 \\ 8 & 5-15 & 10 \\ 12 & 8-20 & 12 \\ 16 & 8-30 & 20 \\ 20 & 16-32 & 25 \\ 24 & 20-60 & 40 \\ 28 & 35-80 & 50 \\ 32 & 40-80 & 60 \\ 36 & 50-100 & 68 \\ 40 & 50-120 & 70\end{array}$

NOTA: Los valores entre nosotros son ligeramente más bajos.

\section{HISTORIAS CLINICAS}

Dividimos las Historias Clínicas recogidas para determinación de Pregnandiol, en tres grupos:

Primer Grupo: Aquellas en las cuales se hizo pronóstico favorable a la gestación, basado en el nivel normal del pregnandiol urinario.

Segundo Grupo Aquellas en las cuales se hizo pronóstico reservado (malo) en cuanto a supervivencia de la gestación, basados en el nivel bajo del pregnandiol urinario.

Tercer Grupo: Corresponde a dos casos de Mola Hidatidiforme. 


\section{HISTORIAS DE PRONOSTICOS FAVORABLE A LA GESTACION}

\begin{tabular}{|c|c|c|c|c|c|c|c|c|}
\hline $\begin{array}{c}\text { Obser- } \\
\text { vacio- } \\
\text { nes }\end{array}$ & Materia Consulta & $\begin{array}{l}\text { Número } \\
\text { Gestac. }\end{array}$ & & $\begin{array}{l}\text { dad } \\
\text { le la } \\
\text { estac. }\end{array}$ & $\begin{array}{l}\text { Miligram. } \\
\text { Pregnan. } \\
24 \text { Horas }\end{array}$ & $\begin{array}{c}\text { Miligram. } \\
\text { Pregnan. } \\
\text { Normal }\end{array}$ & Control & Resultado \\
\hline 1 & Parto prematuro... & $6^{\mathrm{a}}$ & 4 & meses & 4.55 & $8-30$ & $9-34$ & Bueno \\
\hline 2 & Amenaza aborto.... & $2^{\mathrm{a}}$ & 3 & meses & 3.19 & $8-30$ & $1-42)$ & \\
\hline & & & & & & & $11-49)$ & Bueno \\
\hline 3 & Amenaza aborto... & $2^{a}$ & 2 & meses & 7.20 & $5-15$ & 1 & Bueno \\
\hline 4 & Amenaza aborto... & $5^{\natural}$ & 4 & meses & 11.76 & $8-30$ & $29-48$ & Bueno \\
\hline 5 & Amenaza aborto.... & $13^{a}$ & $4^{1 / 2}$ & meses & 24.81 & $10-30$ & - & Bueno \\
\hline 6 & Aborto anterior..... & $3 \mathrm{a}$ & 2 & meses & 3.96 & $5-15$ & $5-47$ & Bueno \\
\hline 7 & Aborto anterior..... & $2 a$ & 1 & mes & 2.47 & $5-15$ & $6-60$ & Bueno \\
\hline 8 & Amenaza aborto.... & $3 a$ & $1 \div 2$ & mes & 0.30 & $5-15$ & $0-881$ & \\
\hline & & & & & & & $1-72$ & \\
\hline & & & & & & & $4-89)$ & Bueno \\
\hline 9 & Amenaza aborto.... & $1^{a}$ & $21^{1}$ & meses & 0.59 & $6-20$ & $18-62$ & Bueno \\
\hline 10 & Parto prematuro... & $3^{\mathrm{a}}$ & 2 & meses & 5.20 & 5-15 & $14-32$ & Bueno \\
\hline 11 & Aborto habitual.... & $6{ }^{\mathrm{a}}$ & 4 & meses & 22.98 & $8-30$ & - & Bueno \\
\hline 12 & Aborto habitual.... & 6 & 2 & meses & 30.98 & $5-15$ & - & Bueno \\
\hline 13 & Amenaza aborto.... & $1 a$ & 4 & meses & 18.68 & $8-30$ & - & Bueno \\
\hline 14 & Amenaza aborto.... & $1^{\mathrm{a}}$ & 3 & meses & 21.00 & $8-30$ & - & Bueno \\
\hline 15 & Amenaza aborto.... & $3 a$ & 2 & meses & 10.08 & $5-15$ & - & Bueno \\
\hline 16 & Aborto anterior.... & 5 & 5 & meses & 1.44 & $16-30$ & $30-40$ & Bueno \\
\hline 17 & Aborto habitual.... & $6^{\mathrm{a}}$ & $11 / 2$ & mes & 5.79 & $5-15$ & - & Bueno \\
\hline 18 & Amenaza aborto.... & $1^{\mathrm{a}}$ & $1^{1} 2$ & mes & 0.94 & $5-15$ & 4.60 & Bueno \\
\hline 19 & Aborto habitual.... & 5 & $1^{1} 2$ & mes & 6.65 & $5-15$ & - & Bueno \\
\hline 20 & Parto prematuro.... & $6 \mathrm{a}$ & 2 & meses & 8.36 & $5-15$ & - & Bueno \\
\hline 21 & Aborto anterior.... & $2^{\mathrm{a}}$ & 1 & mes & 8.85 & $5-15$ & - & Bueno \\
\hline 22 & Aborto anterior.... & $6^{a}$ & 2 & meses & 3.40 & $5-15$ & - & Bueno \\
\hline 23 & Amenaza aborto.... & $1 a$ & $1 \frac{1}{2}$ & mes & 0.39 & $5-15$ & - & Bueno \\
\hline 24 & Amenaza aborto.... & $5^{a}$ & 4 & meses & 0.39 & $8-30$ & - & Bueno \\
\hline 25 & Amenaza aborto.... & $1 \mathrm{a}$ & $11 / 2$ & mes & 1.98 & $5-15$ & $0-42$ & Bueno \\
\hline 26 & Amenaza aborto.... & $2^{\mathrm{a}}$ & 2 & meses & 1.62 & $5-15$ & 3.78 & Bueno \\
\hline 27 & Amenaza aborto.... & $6^{3}$ & $11 / 2$ & mes & 1.18 & $5-15$ & 2.29 & Bueno \\
\hline 28 & Amenaza aborto.... & 19 & 2 & meses & 9.73 & $5-15$ & - & Bueno \\
\hline 29 & Amenaza aborto.... & $5^{\mathrm{a}}$ & 3 & meses & 0.50 & $8-20$ & $24-75$ & Bueno \\
\hline 30 & Amenaza aborto.... & 19 & 4 & meses & 14.96 & $8-30$ & - & Bueno \\
\hline
\end{tabular}

\section{HISTORIAS DE PRONOSTICO DESFAVORABIE A LA GESTACION}

\begin{tabular}{|c|c|c|c|c|c|c|c|c|}
\hline $\begin{array}{c}\text { Obser- } \\
\text { vacio- } \\
\text { nes }\end{array}$ & Materia Consulta & $\begin{array}{l}\text { Número } \\
\text { Gestac. }\end{array}$ & & $\begin{array}{l}\text { dad } \\
\text { e la } \\
\text { estac. }\end{array}$ & $\begin{array}{l}\text { Miligram. } \\
\text { Pregnan. } \\
24 \text { Horas }\end{array}$ & $\begin{array}{c}\text { Miligram. } \\
\text { Pregnan. } \\
\text { Normal }\end{array}$ & Control & Resultado \\
\hline 了 & Varias $\ldots \ldots \ldots \ldots$. & $7^{\mathrm{a}}$ & 1 & mes & 2.54 & $5-15$ & $2-44$ & Abortó \\
\hline 2 & Aborto habitual... & $6^{\mathrm{a}}$ & 3 & mes & 2.87 & $8-20$ & $1-92$ & " \\
\hline 3 & Amenaza aborto... & $1^{\mathrm{a}}$ & $31 / 2$ & mes & 44.80 & $8-20$ & $10-15$ & " \\
\hline 4 & Amenaza aborto... & $1 \mathrm{a}$ & 1 & mes & 1.32 & $5-15$ & $0-54$ & 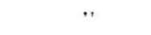 \\
\hline 5 & Aborto habitual... & $9 a$ & 1 & mes & 3.22 & $5-15$ & $0-5$ & ". \\
\hline 6 & Amenaza aborto... & $3^{\mathrm{a}}$ & $2: 2$ & meses & 3.48 & $5-15$ & $2-22$ & ". \\
\hline 7 & Amenaza aborto.... & $7^{\mathrm{a}}$ & 4 & meses & 0.39 & $8-30$ & - & .. \\
\hline 8 & Amenaza aborto... & $5^{\mathrm{a}}$ & 21,2 & meses & 1.81 & $5-15$ & - & .. \\
\hline 9 & Amenaza aborto... & $7^{4}$ & 3 & meses & 0.93 & $8-20$ & - & ". \\
\hline 10 & Amenaza aborto... & $1^{\mathrm{a}}$ & $3 \frac{1}{2}$ & meses & 3.16 & $8-20$ & - & ." \\
\hline 11 & Amenaza aborto... & $12^{a}$ & 4 & meses & 3.96 & $8-30$ & $2-+47$ & No aborto \\
\hline 12 & Amenaza aborto... & 14 & 31 & meses & $2: 54$ & $8-24$ & $\ldots$ & Aborio \\
\hline 13 & Amenaza aborto... & $1 \stackrel{4}{4}$ & 2 & meses & 1.37 & $5-15$ & - & . \\
\hline
\end{tabular}




\section{MOLAS HIDATIDIFORMES}

\begin{tabular}{|c|c|c|c|c|c|c|c|}
\hline $\begin{array}{c}\text { Obser- } \\
\text { vacio- } \\
\text { nes }\end{array}$ & Materia Consulta & $\begin{array}{l}\text { Número } \\
\text { Gestac. }\end{array}$ & $\begin{array}{l}\text { Edad } \\
\text { de la } \\
\text { Gestac. }\end{array}$ & $\begin{array}{l}\text { Miligram. } \\
\text { Pregnan. } \\
24 \text { Horas }\end{array}$ & $\begin{array}{c}\text { Miligram. } \\
\text { Pregnan. } \\
\text { Normal }\end{array}$ & Control & Resultado \\
\hline 1 & Amenaza de & $2^{\mathrm{a}}$ & $3 \mathrm{~m}$ & 1.22 & $8-20$ & - & M \\
\hline 2 & Amenaza de aborto. & $3^{\mathrm{a}}$ & 3 meses & 2.00 & $8-20$ & - & Mola Hidatid. \\
\hline
\end{tabular}

\section{RESUMEN}

Los datos obtenidos en las historias anteriores nos llevaron a concluír que el único sistema o método práctico y rápido para pronosticar el éxito o el fracaso de un aborto amenazante es la estimación cuantitativa del pregnandiol urinario. Es evidente que la mayor parte de las pacientes con aborto amenazante y con una baja excreción de pregnandiol y, particularmente en aquellas en quienes un descenso progresivo y rápido en la mencionada excreción de pregnandiol, e] pronóstico o concepto en cuanto a supervivencia de la gestación es no viable. Por otra parte, en la mayoria de las pacientes cuando el tiempo de los cólicos uterinos y de las sangrías llega, ya es, o puede ser, muy tarde para evitar el aborto. Contrariamente, cuando la excreción de pregnandiol permanece normal, nosotros creemos que es una buena evidencia para fundamentar el concepto de viable; en tal caso debe hacerse todo esfuezo terapéutico posible para conservar la preñez.

Los americanos aconsejan que la dosis útil para terapéutica hormonal, debe ser alta cuando el pregnandiol es bajo, y que debe ser inferior a 100 miligramos diarios de progesterona, pues por experimentos que han realizado manifiestan que solamente el $10 \%$ de progesterona administrada es la que se utiliza.

No tenemos suficiente experiencia en este tópico y dimos solamente dosis de 20 miligramos, como máximo.

En deficiencia notoria de pregnandiol urinario deben cmplearse dosis altas de progesterona de la misma manera que en los casos de buena eliminación de pregnandiol urinario, no es necesario administrar dicha hormona.

Reunimos un total de 45 observaciones. A varias de esas pacientes les dosificamos por varias veces la eliminación de pregnandiol, de manera que, en los cuatro resúmenes expuestos, llegamos a un total de 69 determinaciones verificadas en el presente trabajo.

Como materia de consulta fueron principalmente: el aborto amenazante, d abonto habitual y partos prematuros antcriores.

En los dos casos de Mola Mlidatidiforme, se encontraron niveles bajos de diminación de pregnandiol urinario.

En cuanto al número de la gestación, vimes desde la primogestante hasta la décimotercera gestación.

En relación al ticmpo de gestación, nos interesio desde el primer mes de amenorrea hasta el quinto mes. 
El bucn pronóstico basado en la eliminación del pregnandiol, si no alta por lo menos normal, se cumplici en el 80\% de los casos.

El mal pronóstico basado en deficiente climinación de pregnandiol urina rio, se cumplio en el $77 \%$ de los casos.

\section{CONCLUSIONES}

$1^{a}$ - La determinación del pregnandiol urinario es de máxima utilidad en los casns de Aborto Amenazante, Aborto Habitual y Partos Prematuros Anteriores.

$2 \%-E l$ 80\% de los casos de amenaza de aborto con buen nivel de eliminación de pregnandiol urinario, se pronosticaron felizmente.

3?-El mal pronóstico basado en deficiente eliminación del pregnandiol urinario, se cumplio en el $77 \%$ de los casos.

4a-En los casos de amenaza de aborto y deficiente eliminación de pregnandiol urinario, son útiles las dosis altas de progesterona como tratamiento. 5:- La determinación cuantitativa del pregnandiol urinario debe ser rutina en los centros de matemidad.

\section{B I B L I O G R A F I A}

1.-R. N. Rutherford: Amer. J. Obst. 51, 652 (1946).

2.-P. Malpas: J. Obst. 45, 932 (1938).

3.-P. M. F. Bishop N. A. Richards: Brit. Med. J. 1950, II, 130.

4.-N. W. Vaux y A. E. Rakoff: Amer. J. Obst. 50, 353 (1945).

5.-M. E. Davis y N. W. Fugo: J. Amer. Med. Assoc. 142, 778 (1950).

6. -Karl Yunkmann: "Hormonas como Medicamentos". - Selecciones- Médica de Colombia, No 4, Bogotá IV. - 53.

7.-Marian, G. F.: Biochem. J. 23: 1090 y 1929.

8.-Venning, Eleanor H. y Browne: J. S. L., Isolation of water soluble pregnane diol complex from human pregnancy urine. Pro. Soc. Exper. Biol., etc. Med. 34; 792/793 (June) 1936

9.-Venning, Eleanor H.; Henry, J. S. and Browne: J. S. L. The Measurement of a pregnanediol complex in human urine. Canad. M. A. J. 36: 83, Jan. 1937. 10.-Arthur K. Koff y Alex Tulsky: The Surgical Clinics of North-America, 1953. 33, Number 1. "La Amenaza de Aborto".

11. - Venning, Eleanor H.: Grarimetric Method for the Determination of Sodium Pregnanediol Glucoronidate (an excretion product of progesterone). J. Biochem. 119; 473-480 (July) 1937.

12. - Gutherman, H. S.: A human pregnancy test based upon a color reaction of pregnanediol in the urine. J. Clin. E. 4:262/267 (June) 1944.

Hoyt, R. E. and Levine, M. G.: Improved quantitative estimation of urinary pregnanediol. J. of Clin. E. 1: 89-107, Jan. 1950.

13. - Valor diagnostico y Pronóstico de la excreción urinaria de pregnandiol, estu diada por el método de Gutherman. - Tesis para optar a título de Técnica de Laboratorio Clínico - Zoila Rey de Hormaza. 1950.

14. - Comptes Rendus de la Société Francaise de Gynecologie. - XXII Année, No 3. Mars. 1952

15. - Sommervilie-Marrian y Kellar: -1948- Lancet 11. - 89.

16.-Cantarwow: Clinical Biochemestry, 1950. 